\title{
Potencial invasor de Syzigium jambos (Myrtaceae) en fragmentos boscosos: El caso de Ciudad Colón, Costa Rica
}

\author{
José Fco. Di Stéfano, Luis A. Fournier, Julieta Carranza, Walter Marín y Adriana Mora ${ }^{1}$ \\ 1 Escuela de Biología, Universidad de Costa Rica, 2060 San José, Costa Rica.
}

Recibido 18-VII-1997. Corregido 24-XI-1998. Aceptado 17-IV-1998.

\begin{abstract}
Syzigium jambos is a multiple use species native to Southeast Asia that has been introduced in many countries of the world. Similar to some other exotics, it has the potential to become naturalized and invade natural ecosystems. In Costa Rica, it has been observed in several small preserves. A sample of 48 quadrats of $0.6 \mathrm{~m}$ of radius, in one hectare fragment (with a 30 year-old secundary humid Premontane forest) had a density of 4.9 seedlings and 0.16 saplings per $\mathrm{m}^{2}$. Higher densities were observed near the base of the adult trees $(\mathrm{N}=3)$ and on the biggest of the trees investigated. Growth rates of the seedlings and saplings was very low: less than 10.5 and $0.25 \mathrm{~cm} /$ year in height and basal diameter, respectively. In general, the plants had low levels $(<30 \%)$ of relative damage by herbivores, and fungi (one identified as a Puccinia psidii Wint.) on their leaves, although a significant increase was detected by the end of the wet season and beginning of the dry season. Small white epiphyllic liquens were abundant on mature leaves. Apparently the species is very flexible as little mechanical damage was observed.
\end{abstract}

Key words: Invasion potential, exotic species, damage, tropical forest fragments.

El intercambio de especies (flujo genético) entre continentes se ha incrementado en los últimos siglos. Por ejemplo, para los archipiélagos de Hawaii, la colonización natural de angiospermas ocurrió a una tasa de una especie/100.000 años, pero con la ocupación humana de las islas, ésta subió a una especie/dos años (Loope et al. 1988, en Macdonald 1994). Las posibilidades de arribo de nuevas especies son aún mayores para los ecosistemas continentales.Varias especies exóticas han desplazado a las nativas al verse favorecidas por la "liberación ecológica" (ésta tiene menores probabilidades de encontrar depredadores y parásitos en el nuevo sitio), y por los altos niveles de alteración o estrés en los ecosistemas donde se establecen (Macdonald 1994).
Algunas de ellas se han convertido en plagas como Melaleuca sp. (Di Stéfano y Fisher 1983) o Schinus sp. (Ewel 1979), en el sur de Florida, Lantana camara L. en las Islas Pacífico (Thaman 1974), Ulex europaeaus L., en las cercanías del Volcán Poás, Costa Rica (Macey 1975), o varias gramíneas africanas introducidas a América Latina (e.g. Pennisetum clandestinum Hochst.).

Las reservas forestales de pocas hectáreas son todavía frecuentes en Costa Rica, y dada su condición de inestabilidad ecológica, pueden ser sitios potenciales para el establecimiento y expansión de especies exóticas. Por ejemplo, en Tabarcia, Ciudad Colón y San Pedro, ocurren Erythrina poeppigiana (Walp)Cook, Coffea 
arabica L., Citrus sp. y Syzigium jambos (L.) Alst. (manzana rosa) tanto en el dosel como en la regeneración (Di Stéfano et al. 1996, Di Stéfano y Morales 1993), ésta última, con un abundante número de plántulas.

$\mathrm{Al}$ respecto, se decidió estudiar la densidad, frecuencia, nivel de daños, supervivencia y crecimiento de $S$. jambos en un bosquete dentro de la zona de vida del Premontano Húmedo (sensu Holdridge 1978), y determinar su valor de importancia.

S. jambos es un árbol de 15 a $20 \mathrm{~m}$ de altura, originario del Sur-Este tropical de Asia. Se ha introducido en muchas regiones del mundo por ser de uso múltiple (Francis 1990, Little, Jr. y Wadsworth 1964). Se utiliza como ornamental, productor de miel de abeja, leña, carbón, postes, medicina, sombra, rompevientos, y cercas vivas. Su fruto es consumido por humanos y el ganado, especialmente cerdos.

\section{MATERIALES Y MÉTODOS}

El estudio se realizó de sẹtiembre de 1995 a agosto de 1996. La reserva forestal de aprox. una hectárea, tiene más de 30 años de estar en regeneración natural. Se encuentra ubicada en Ciudad Colón de Mora, Costa Rica, $\left(84^{\circ} 15^{\prime} \mathrm{W}\right.$, $9^{\circ} 55^{\prime} \mathrm{N}$ ), a $800 \mathrm{msnm}$. La temperatura y precipitación total anual promedio son de $23.6 \mathrm{C}$ y $2050 \mathrm{~mm}$, respectivamente. Durante el estudio se registró una mayor cantidad de lluvia (Fig. 1, L.A. Fournier, datos no publicados tomados in situ). La altura promedio del dosel es de unos $20 \mathrm{~m}$, con dos estratos. Los suelos son coluvioaluviales de muy buena fertilidad.

Se escogieron tres árboles (Cuadro 1) de $S$. jambos separados al menos $20 \mathrm{~m}$. El individuo con el alto DAP se debió a la sumatoria de más de 20 tallos de rebrote. Éste estaba en el lote antes de que se estableciera la reserva.

Para calcular la densidad de las plántulas con respecto a la distancia de la base del árbol, se establecieron un total de 48 parcelas de 0.6 $\mathrm{m}$ de radio en cuatro direcciones a $90^{\circ}$ una de otra, a uno (100\% bajo la copa), cuatro ( $8 \%$ bajo, $67 \%$ en el borde y $25 \%$ fuera de la copa), ocho y $12 \mathrm{~m}$ (todas fuera de la copa). Se hizo un análisis de varianza con los datos transformados a la raíz cuadrada de la densidad +0.5 .

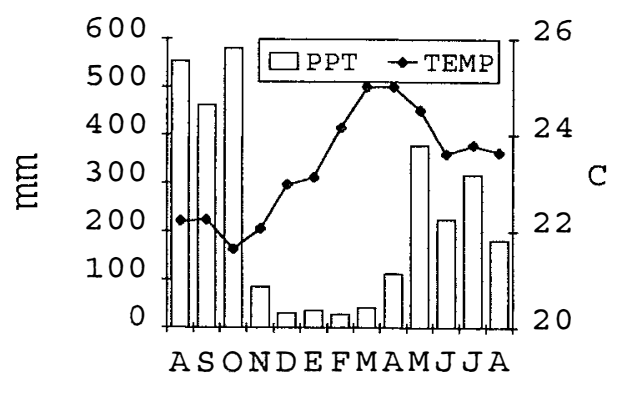

MES

Fig. 1. Precipitación y temperatura promedio durante 19951996, Ciudad Colón, Costa Rica.

\section{CUADRO 1}

Características de los árboles de S. jambos utilizados en el el estudio, Ciudad Colón de Mora, Costa Rica.

Arbol No. Ht (m) DC (m) AC (m²) DAP (cm)

$\begin{array}{ccccc}1 & 8 & 4.4 & 16 & 15.7 \\ 2 & 8 & 7.9 & 49 & 10.9 \\ 3 & 12 & 9.7 & 74 & 139.0\end{array}$

$\mathrm{Ht}=$ altura. $\mathrm{DC}=$ diámetro de copa (promedio de dos direcciones). $\mathrm{AC}=$ área promedio de cobertura de las copas. $\mathrm{DAP}=$ diámetro a la altura del pecho $(1.3 \mathrm{~m})$.

Además, se marcaron cinco puntos distribuidos uniformemente alrededor de tres anillos a 1,4 y 8 $\mathrm{m}$ de la base de cada uno de los árboles. En cada uno de los ellos se escogieron tres plantas, las más cercanas alrededor del mismo a las cuales se les registró, a intervalos de uno o dos meses, la altura a la yema apical, diamétro basal y los aparentes daños ocasionados por hongos (manchas redondeadas de color café en las hojas), herbívoros (principalmente por cortadores, aunque no siempre fue clara la distinción entre las aperturas dejadas por la caída de tejido necrosado por el hongo, y aquéllas por insectos), mecánicos (quebraduras y hojas dobladas o deformadas), líquenes, y número de 
yemas dañadas (una vez al finalizar el experimento), tanto en plántulas como juveniles.

La estimación de los daños fue cualitativa en una escala de 0 a 5 ( 0 indicó ausencia del daño), con valoraciones decimales. Los datos se transformaron a los porcentajes correspondientes y se analizaron con la prueba de Kruskal-Wallis donde los anillos fueron el factor de agrupación.

En marzo de 1996 se establecieron 10 cuadrículas de $100 \mathrm{~m}^{2}$ dentro del bosquete, colocando internamente, una de $25 \mathrm{~m}^{2}$, y tres de 1 $\mathrm{m}^{2}$. Algunas de ellas incluyeron S.jambos marcados anteriormente. Éstas se utilizaron para calcular el índice de valor de importancia (sumatoria de la densidad, frecuencia y área basal relativas/3). En mayo de ese año, se estimaron los daños en las mismas categorías y escala anotadas anteriormente.

\section{RESULTADOS}

$S$. jambos mostró un valor de importancia muy alto tanto para juveniles como plántulas (Cuadro 2). La densidad promedio fue de $0.16 \mathrm{y}$ $4.9 \mathrm{ind} / \mathrm{m}^{2}$, respectivamente, y la especie apareció en más de un $70 \%$ de las parcelas. (En la categoría de árboles ocupó el onceavo lugar.)

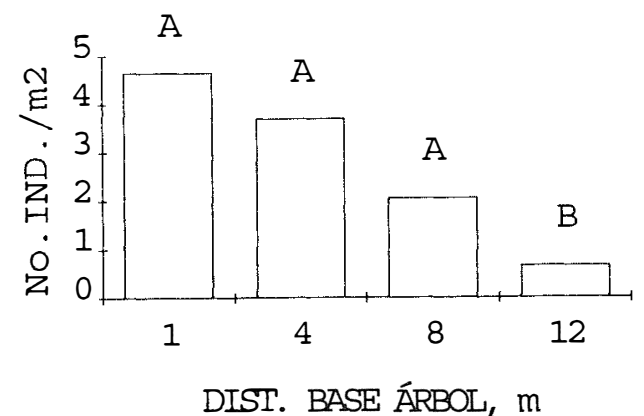

Fig. 2. Densidad promedio de plántulas y juveniles de $S$. jambos con respecto a la distancia de la base del árbol adulto. Letras diferentes indican significancia con $\mathrm{p}$ Bonferroni $<0.05$.

\section{CUADRO 2}

Primeras tres especies de acuerdo al índice de importancia (IVI) en una pequeña reserva forestal, Ciudad Colón, Costa Rica.

$\begin{array}{llllll}\text { Familia } & \text { Especie } & \text { Ht m } & \text { Diam } \mathrm{cm} & \text { Dens.Tot. Frec. Ab.\% } & \text { IVI \% }\end{array}$

Plántulas (<1.5 m ht). n=30. Parcelas de $1 \mathrm{~m}^{2}$.

$\begin{array}{ccccccc}\text { Myrtaceae } & \text { Syzigium jambos } & 21 & 42 & 147 & 77 & 44 \\ \text { Apocynaceae } & \begin{array}{c}\text { Stemmadenia donnell- } \\ \text { smithii (Rose) Woodson }\end{array} & 8 & 13 & 33 & 53 & 27 \\ \text { Sapindaceae } & \begin{array}{c}\text { Cupania guatemalensis } \\ \text { (Turcz) Radlk. }\end{array} & 3 & 8 & 24 & 53 & 20\end{array}$

Juveniles (1.5 m ht $\mathrm{y}<5 \mathrm{~cm}$ DAP). $\mathrm{n}=10$. Parcelas de $25 \mathrm{~m}^{2}$.

$\begin{array}{lcccccc}\text { Myrtaceae } & \text { S. jambos } & 105 & 53 & 41 & 70 & 86 \\ \text { Lauraceae } & \begin{array}{c}\text { Cinnamomum brenesii } \\ \text { (Standl.) Comb. ined. }\end{array} & 40 & 25 & 13 & 60 & 27 \\ \text { Meliaceae } & \text { Trichilia havanensis Jacq. } & 27 & 16 & 11 & 70 & 20\end{array}$

Adultos. (5 cm DAP). $\mathrm{n}=10$. Parcelas de $100 \mathrm{~m}^{2}$.

$\begin{array}{ccccccc}\text { Fabaceae } & \text { Lonchocarpus salvadorensis Pittier } & 84 & 262 & 5 & 40 & 43 \\ \text { Apocynaceae } & \text { S. donnell-smithii } & 185 & 208 & 16 & 50 & 40 \\ \text { Fabaceae } & \text { Machaerium biovulatum Mich. } & 123 & 119 & 9 & 60 & 21\end{array}$


En general, la densidad disminuyó al alejarse de la base del árbol adulto (Fig. 2), y la variabilidad espacial fue alta (coeficientes de variación $>100 \%$ ), encontrándose la mayor abundancia alrededor del árbol más viejo. Esto se debe al traslape de varios cohortes y a una posible mayor producción de frutos.

Los individuos más altos y gruesos también ocurrieron con mayor frecuencia en las cercanías del árbol viejo, pero hubo diferencias significativas ( $<<0.01$ ) entre anillos (Figs. 3 y 4). Las plantas más pequeñas se encontraron a $1 \mathrm{~m}$ de la base.

Luego de 356 días, las plantas a 1,4 y $8 \mathrm{~m}$ de la base habían crecido, en promedio, 7.6, 10.5, y

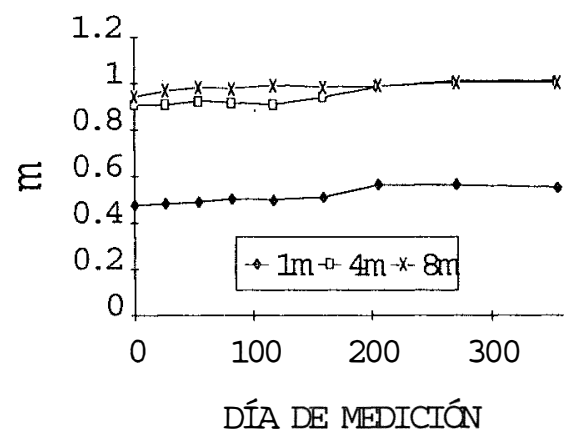

Fig. 3. Altura promedio de plántulas y juveniles de $S$. jambos a 1,4 y $8 \mathrm{~m}$ de las bases de los árboles adultos, Ciudad Colón, Costa Rica, de setiembre 1995 a agosto 1996. Coeficientes de variación entre un 60 y $95 \%$, siendo menores en el anillo a $8 \mathrm{~m}$.

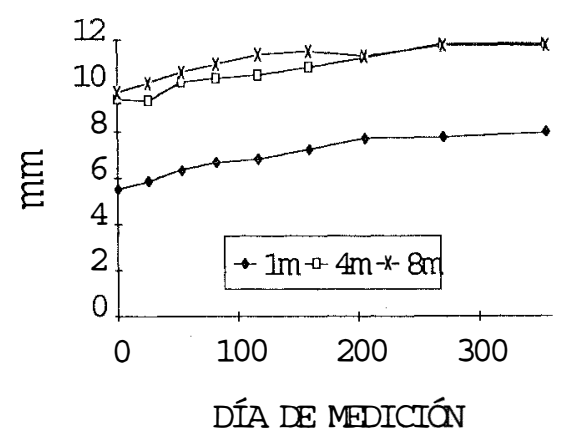

Fig. 4. Diámetro basal promedio de plántulas y juveniles de $S$. jambos a 1, 4 y $8 \mathrm{~m}$ de las bases de los árboles adultos, Ciudad Colón, Costa Rica, de setiembre 1995 a agosto 1996. Coeficientes de variación entre un 40 y $75 \%$, siendo menores en el anillo a $8 \mathrm{~m}$.
$6.2 \mathrm{~cm}$ en altura, y $2.45,2.44$ y $2.04 \mathrm{~mm}$ en diámetro basal, respectivamente. Las primeras, a su vez, mostraron la mayor mortalidad (17.8\%) comparado con 11.1 y $0 \%$ en los individuos a 4 y $8 \mathrm{~m}$.

En general, las plantas aparecieron con daños relativos menores al $30 \%$ en la mayoría de las fechas (Figs. 5-8). Sin embargo, hubo un aparente incremento a finales de la época lluviosa y principios de la seca, especialmente en las plantas más alejadas de la base del árbol (diferencias significativas entre anillos de noviembre a enero $(\mathrm{p}<0.05)$ para los hongos, y de octubre a febrero para los líquenes ( $\mathrm{p}<0.02)$, no así para la herbivoría).

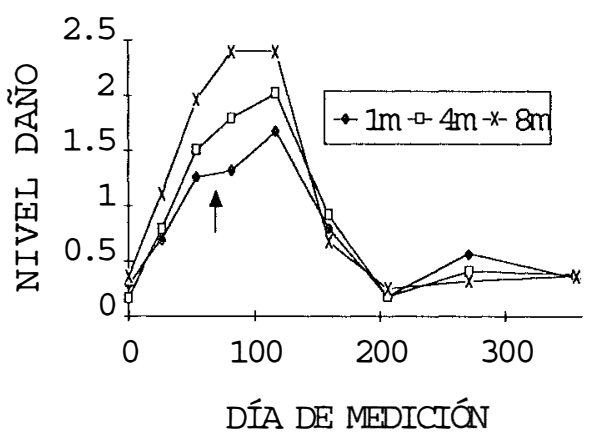

Fig. 5. Daños relativos por hongos (escala cualitativa de 0 a 5) en las hojas de plántulas y juveniles de $S$. jambos a tres distancias de la base del árbol adulto, Ciudad Colón, Costa Rica, de setiembre 1995 a agosto 1996. Coeficientes de variación entre un 30 y $180 \%$. Flecha indica inicio de la época seca (finales de noviembre-diciembre).

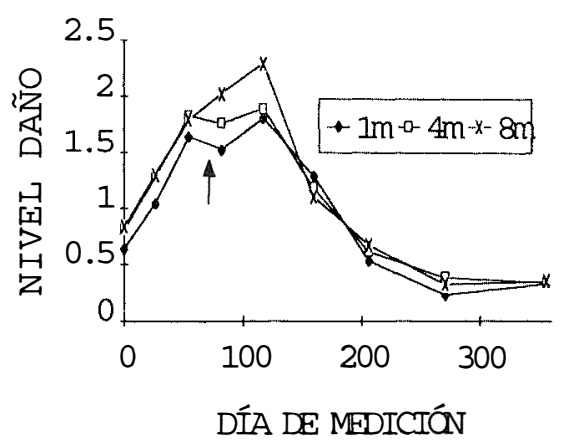

Fig. 6. Daños relativos causados por herbívoros (escala cualitativa de 0 a 5) en hojas de plántulas y juveniles de $S$. jambos a tres distancias de la base del árbol adulto, Ciudad Colón, Costa Rica de setiembre 1995 a agosto 1996. Coeficientes de variación entre un 30 y $100 \%$. Flecha indica inicio de la época seca. 


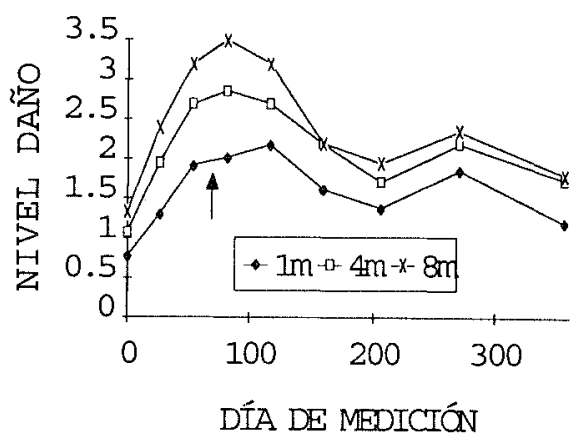

Fig. 7. Presencia relativa de líquenes sobre las hojas (escala cuali ativa de 0 a 5 ) en plántulas y juveniles de $S$. jambos a res distancias de la base del árbol adulto, Ciudad Colón, Costa Rica. Coeficientes de variación entre un 25 y $115 \%$. Flecha indica inicio de la época seca.

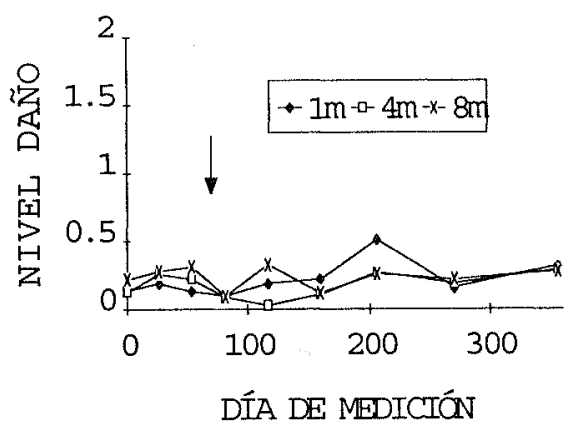

Fig. 8. Daños mecánicos relativos (escala cualititativa de 0 a 5) en plántulas y juveniles de $S$. jambos a tres distancias de la base del árbol adulto, Ciudad Colón, Costa Rica, de setiembre 1995 a agosto de 1996. Coeficientes de variación entre un 100 y $500 \%$. Flecha indica inicio de la época seca.

Los daños mecánicos fueron muy bajos (Fig. 8). Se determinó que las plántulas y juveniles son muy flexibles y aparentemente logran absorber los golpes producto de la caída de ramas o el pizoteo (con frecuencia los alambres usados para marcar las plántulas estaban doblados, mientras que a las plántulas no se les detectaba quebraduras).

$\mathrm{Al}$ analizar los daños en las parcelas de $1 \mathrm{~m}^{2}$ en mayo de 1996 (al inicio de la época lluviosa), se determinó que las plántulas de $S$. jambos presentaban una menor probabilidad de encontrarlas con daños mecánicos, una mayor de que tuvieran hongos en sus hojas, y similar herbivoría cuando se compararon con las especies nativas (Cuadro 3). Por otro lado, a un $8 \%$ de las plántulas se les observó con no más de una yema dañada, mientras que el $22 \%$ de las especies nativas tenían una o más yemas dañadas.

\section{CUADRO 3}

Frecuencia (\%) de las plántulas de S. jambos y especies nativas con daños, según categoría (clase 5 , más alto), en un fragmento forestal, Ciudad Colón, Costa Rica. Mayo de 1996.

\begin{tabular}{ccccccc} 
Sp./Clase & 0 & $<1$ & $<2$ & $<3$ & $<4$ & $<5$ \\
\multicolumn{7}{c}{ Mecánico } \\
S.jambos & 66 & 34 & 0 & 0 & 0 & 0 \\
Nativas & 57 & 25 & 7 & 9 & 2 & 0 \\
Songos \\
S.jambos & 27 & 68 & 5 & 0 & 0 & 0 \\
Nativas & 72 & $\begin{array}{c}\text { 19 } \\
\text { Herbivoría }\end{array}$ \\
S.jambos & 32 & 60 & 8 & 0 & 0 & 0 \\
Nativas & 28 & 61 & 7 & 3 & 1 & 0
\end{tabular}

\section{DISCUSIÓN}

En general, $S$. jambos presentó una elevada densidad de individuos en el sotobosque. Se estimó que, dentro del bosquete, pueden haber más de 40.000 plántulas y 1.600 juveniles. $\mathrm{Al}$ respecto, Little y Wadsworth (1964), y Francis (1990) mencionan que en Puerto Rico la especie se comporta como "maleza", formando densas plantaciones muy difíciles de erradicar. Por otra parte, Aide et al. (1996) determinaron que la especie apareció en más de la mitad de 30 sitios en regeneración estudiados en Puerto Rico, y en un bosque con 60 años, llegó a ser la especie dominante.

La mayor cantidad de plantas ocurrió cerca del árbol adulto (especialmente en el más viejo), pero se encontró una alta varialbilidad en la distribución de las mismas. Con frecuencia se hallaron pequeños parches con una alta concentración de individuos. Si bien la mayoría de los frutos cae bajo la copa (ver Morton 1987 y Francis 1990), se observó comederos de aves y pequeños mamíferos (e.g. ardillas) a cierta distancia del árbol. Además, se conoce que Syzigium es poliembriónica 
produciendo hasta cuatro embriones por semilla (Francis 1990, Morton 1987).

Similar a lo que predice el modelo JansenConnell y como se ha detectado en varias especies arbóreas (Oliveria et al. 1996), la mayor mortalidad de plantas ocurrió cerca del árbol adulto. Francis (1990) señala que las semillas necesitan germinar bajo la sombra, pero posteriormente requieren una apertura en el dosel para desarrollarse. Esto resulta difícil bajo la copa de $S$. jambos dado que ésta es muy cerrada y a que la especie es perennifolia. Además, se observó que las plántulas más pequeñas fueron las más propensas a sufrir estrés hídrico durante la época seca (esta especie es muy sensible a las sequías, Fransis 1990). Sin embargo, no se logró detectar mayores porcentajes de daños en este grupo de plantas.

Las tasas de crecimiento en altura y diámetro dentro del bosquete fueron muy bajos, inferiores a los encontradas por Francis (1990) en Puerto Rico en condiciones de invernadero. Estos últimos crecieron $24 \mathrm{~cm}$ en altura en 250 días.

El porcentaje de daños en general fue menor al $30 \%$. Francis (1990) y Morton (1987) señalan que en Puerto Rico $S$. jambos tienen pocos enemigos naturales y no le causan mayores daños. Sin embargo, se observaron periodos del año donde ios niveles aumentaron considerablemente. Filip et al. (1995) también determinaron variaciones anuales en las tasas de herbivoría, aunque señalan que fue más intensa durante el primer periodo de las lluvias. Para el caso de los hongos, la segunda etapa puedo resultar la más favorable debido a que en este periodo la humedad fue muy alta (Fig. 1). La posterior disminución en el tiempo pudo deberse a la pérdida de hojas dañadas, a un efecto de "dilución" por la aparición de hojas juveniles inicialmente sanas, o ambos.

Para el presente estudio, los líquenes costrosos epífilos sobre hojas maduras (algunas formando pequeñas áreas necrosadas alrededor), fueron los más evidentes, seguido por ataques de hongos (el principal causado por la roya Puccinia psidii Wint., el mismo detectado en Brasil, Fransis 1990), y la herbivoría. Otras investigaciones han informado de ataques por Asterinella y Glomerella (Ascomicetes), y Colletotrichum y Phyllosticta (Deuteromicetes) en Estados Unidos (Farr et al. 1990) y de la presencia de manchas negruzcas en las hojas causadas por áfidos y algas (Morton 1987).

¿Cuáles pueden ser algunos factores que favorecieron a que la especie presente tal agresividad? Primeramente, el sitio en regeneración natural, posee suelos de buena fertilidad y precipitaciones mayores de $1700 \mathrm{~mm}$, los cuales se han señalado como condiciones básicas para la superviencia de la especie (Francis 1990). Segundo, $S$. jambos produce una abundante cantidad de frutos muy apetecidos por la fauna silvestre, en donde la semilla, de aprox. $1 \mathrm{~cm}$ de diámetro, queda libre dentro del pericarpo (se reduce así, la probabilidad de que sea dañada por el frugívoro). Ésta cuenta con buenas reservas nutritivas y energéticas, y una vez germinada, la plántula es tolerante a la sombra y a la competencia, inclusive de hérbaceas (Francis 1990). Desarrolla una raíz muy profusa con alta capacidad de acumular reservas (Francis 1990), y las plántulas y juveniles son muy flexibles, poco propensas a daños mecánicos, capaces de rebrotar en caso de quebrarse. Una vez adulta, produce una densa sombra que impide el crecimiento de otras especies. Además, aparentemente tiene altas concentraciones de taninos la cual puede reducir los daños por herbívoros, y ser alelopática. Al respecto, Céspedes (1986) observó que el follaje de manzana rosa y del café fueron los menos consumidos, al estudiar las preferencias alimenticias del lepidóptero Dirphiopsis flora (Schaus) en el laboratorio.

Como posibles limitantes para la especie en el sitio, fueron sus muy bajas tasas de crecimiento, su alta sensibilidad a la sequía, y los moderados a altos niveles de daños foliares por el hongo Puccinia psidii y el liquen costroso.

Para este bosquete, donde el objetivo principal es favorecer la mayor diversidad de especies vegetales nativas, se recomienda eliminar paulatinamente tanto los adultos (anillando y controlando los retoños) como la regeneración (arrancándolas para no fomentar el rebrote, ver Francis 1990). Con ello, se formarían pequeños 
claros y espacios libres los cuales pueden ser aprovechados por las plantas nativas.

\section{RESUMEN}

Syzigium jambos es una especie de uso múltiple originaria del Sur-Este de Asia, la cual ha sido introducida en muchos países del mundo. Asi como algunas otras exóticas, tiene el potencial de naturalizarse y de invadir ecosistemas naturales. En Costa Rica, se ha observado en varios fragmentos boscosos. Se establecieron 48 parcelas de $0.6 \mathrm{~m}$ en una hectárea de bosque secuendario de 30 años. Pertenece a la zona de vida del Premontano húmedo. Se estimó que la especie exótica alcanzó una densidad de 4.9 plántulas y 0.16 juveniles por $\mathrm{m}^{2}$. La mayor densidad se observó cerca de la base del árbol adulto y el árbol más grueso. El crecimiento de las plantas fue muy bajo con tasas anuales menores a 10.5 y $0.25 \mathrm{~cm}$ en altura y diámetro basal, respectivamente. En general, se observaron daños relativos menores al $30 \%$ aunque se registró un aumento importante al finalizar la época lluviosa y el inicio de la seca. Las hojas maduras presentaron una abundante cantidad de manchas blaquecinas pequeñas producto de un líquen costroso epífilo. Aparentemente la planta es muy flexible puesto que los daños mecánicos fueron muy bajos.

\section{REFERENCIAS}

Aide, T.M., J.K. Zimmerman, M. Rosario \& H. Marcano. 1996. Forest recovery in abandoned cattle pastures along an elevational gradient in Northeastern Puerto Rico. Biotropica 28: 537-548.

Céspedes, R. 1986. Ecología de Dirphiopsis flora (Schaus, Lepidoptera: Saturniidae) e impacto sobre Quercus aff. seemannii Liebm. en encinares naturales de Costa Rica. Tesis Licenciatura, Universidad de Costa Rica, Costa Rica. 77p.

Di Stéfano, J.F. \& C.O. Morales. 1993. Inventario florístico en varias áreas boscosas en Tabarcia de Mora y Palmichal de Acosta, Costa Rica. Rev. Biol. Trop. 41: 423-431.

Di Stefano, J.F. \& R.F. Fisher. 1983. Invasion potential of Melaleuca quinquenervia in Southern Florida, U.S.A. Forest Ecol.\& Manag. 7: 133-141.

Di Stefano, J.F., V. Nielsen, J.Hoomans \& L.A. Fournier. 1996. Regeneración de la vegetación arbórea en una pequeña reserva forestal urbana del nivel premontano húmedo, Costa Rica. Rev. Biol. Trop. 44: 575-580.

Ewel, J. 1979. Seed dynamics in Shinus in the successional ecosystems of Everglades National Park. Fourth Quarterly Report. Research Center, Everglades National Park. 67p.

Farr, D.F., G.F. Bills, G.P. Chamuris \& A.Y. Rossman. 1989 Fungi on plants and plant products in the U.S. APS, Saint Paul, Minnesota. 1252p.

Filip, V., R. Dirzo, J.M. Maass \& J. Sarukhán. 1995. Withinand among-year variation in the levels of herbivory on the foliage of trees from a Mexican tropical deciduous forest. Biotropica 27: 78-86.

Francis, J.K. 1990. Syzygium jambos (L.)Alst. Rose apple. SO-ITF-SM No. 26, Institute of Tropical Forestry, Southern Forest Exp. Station, USDA, Puerto Rico. 4 p.

Holdridge, L. 1978. Ecología basada en zonas de vida. IICA, San Jose, Costa Rica. 216 p.

Little, Jr., E.L. \& F.H. Wadsworth. 1964. Common trees of Puerto Rico and Virgin Islands. Agric. Handbook No. 249. U.S. Dept. of Agriculture, Forest Service, Washington, D.C. 548p.

Loope, L.L., O. Hamann \& C.P. Stone. 1988. Comparative conservation biology of oceanic archipielagos. Bioscience 38: 272.

Macdonald, I.A. 1994. Global change and alien invasions: Implications for biodiversity and protected area management. p. 199-210. In O.T. Solbrig, H.M. van Emden\& P.G. van Oordt (eds.) Biodiversity and Global Change. CAB International, Oxon, Gran Bretaña.

Macey, A. 1975. The vegetation of Volcán Poás National Park, Costa Rica. Rev. Biol. Trop. 23: 239-255.

Morton, J.F. 1987. Fruits of warm climates. Fl. Dpt. of Agric. \& Consumers Service, Miami, Fl. 505p.

Oliveira-Filho, A.T., A.A. Camisão-Neto \& M.M.L. Volpato. 1996. Structure and dispersion of four tree populations in an area of Montane semideciduous forest in Southeastern Brazil. Biotropica 28: 762-769.

Thaman, R. 1974. Lantana camara: Its introduction, dispersal, and impact on islands of the tropical Pacific Ocean. Micronesia 10: 17-39. 